\title{
Synthesis and Antimicrobial Activity of 3-Cyano-4-imino-9-methoxy-4H-pyrimido $[2,1-b]$ pyrimido $[4,5-b]$ quinoline and 2-Substituted Derivatives
}

\author{
S. P. Vartale, N. K. Halikar, and Y. D. Pawar \\ Department of Chemistry, P.G. Research Centre, Yeshwant Mahavidyalaya, Nanded 431602, India \\ Correspondence should be addressed to S. P. Vartale; spvartale@gmail.com
}

Received 9 December 2011; Revised 30 April 2012; Accepted 14 May 2012

Academic Editor: Filomena Conforti

Copyright (C) 2013 S. P. Vartale et al. This is an open access article distributed under the Creative Commons Attribution License, which permits unrestricted use, distribution, and reproduction in any medium, provided the original work is properly cited.

The 2-amino-7-methoxypyrimido[4,5-b]quinoline (1) on treatment with bis(methylthio)methylene malononitrile (2) in ethyl alcohol and catalytic amount of TEA gives 3-cyano-4-imino-9-methoxy-2-methythio-4H-pyrimido[2,1-b]pyrimido[4,5-b]quinoline (3). The latter were further reacted with selected $\mathrm{N}-, \mathrm{O}-$, and $\mathrm{C}$-nucleophiles such as aryl amines, hetryl amines, substituted phenols, and compounds containing an active methylene group.

\section{Introduction}

Quinoline derivatives represent the major class of heterocycles and a number of preparations have been known since the late 1800s. The quinoline ring system occurs in various natural products, especially in alkaloids. The quinoline skeleton is often used for the design of many synthetic compounds with diverse pharmacological properties. In 1820 , quinine was isolated as the active ingredient from the bark of cinchona tree and successively replaced the crude bark for the treatment of malaria. Despite its relatively low efficacy and tolerability, quinine still plays an important role in the treatment of multiresistant malaria. This molecule has also played a historical role in organic chemistry as a target for structural determination and total synthesis [1] and recently both stereoselective [2] and enantioselective [3] total syntheses. Quinoline and its derivatives are receiving important due to their wide range of biological activities as a drug analgesics [4], antiamoebic [5-8], trypanocidal [9], antiseptic [10], and antiserotonin [11]. In addition to these, derivatives also exhibit good antimalarial [12, 13], antitubercular [14], antibacterial [15], antihistaminic [16], anti-neurodegerneative [17], anticonvulsant [18], antitumor [19], anticancers [20, 21], and antiallergics [22] activities.
In the light of these valid observations, such fused quinoline with pyrimidine ring would exhibit some interesting pharmacological activities; further, the ring anellation to amino groups containing nitrogen heterocycles with ketene dithioacetals as reagent has been reported [23$25]$. Recently, we report one pot synthesis of 3-cyano9-methyl-2-methylthio-4-oxo-4H-pyrimido [2,1-b] pyrimido $[4,5-b]$ quinoline and its reaction with selected nucleophiles [26]. In continuation, this remains an opportunity for further development of milder condition with better yields. The compound 3 was prepared by the reaction of 2-amino-7-methoxy pyrimido [4,5-b] quinolone 1 with bis(methylyhio)methylene malononitrile 2 in presence of ethyl alcohol and catalytic amount of TEA, Scheme 1. A plausible mechanism for the formation of parent compound 3 can be adduced as shown in Scheme 2. Compound 3 possesses an active methylthio group at the 2 positation that is activated by the ring 1-nitrogen atom and the electron withdrawing 3 -cyano group. Compound 3 was reacted with selected N-, O-, and C-nucleophiles like aryl amines, substituted phenols, heteryl amines, and compound containing active methylene group. Hence, compound 3 independently reaction with different substituted anilines, substituted phenols, active methylene compounds, and hetryl amines in presence of 
<smiles>COc1ccc2nc3nc([NH3+])ncc3cc2c1</smiles><smiles>CC(C)=C(C)C#N</smiles>

2<smiles>COc1ccc2nc3c(cnc4nc(SC)c(C#N)c(=N)n43)cc2c1</smiles>

3

ii) $\mathrm{EtOH} / \mathrm{TEA}$

SCHEMe 1<smiles>COc1ccc2nc3nc([NH])ncc3cc2c1</smiles>

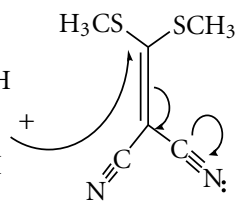

2<smiles>COc1ccc(I)c(C)c1</smiles>

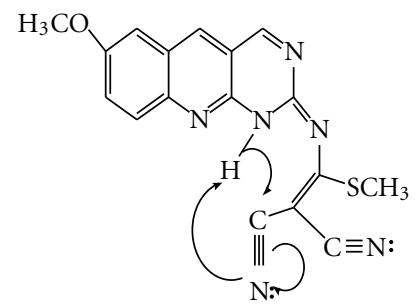<smiles>C1CCCC1</smiles>

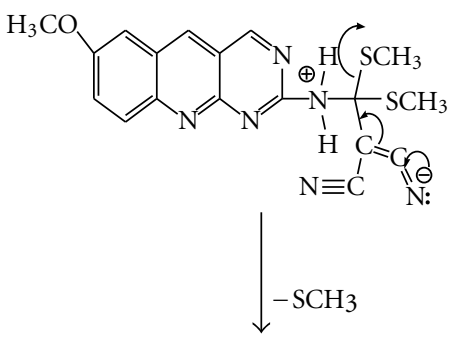

$\mathrm{H}_{3} \mathrm{CO}$<smiles>CC(C#N)C(C#N)C([Hg])Nc1ncc2cc3ccccc3nc2n1</smiles><smiles>COc1ccc2nc3c(cnc4nc(C)c(C#N)c(=N)n43)cc2c1</smiles>

3

Scheme 2: Mechanism: plausible mechanism 3-cyano-4-imino-9-methoxy-2-methythio-4H-pyrimido [2,1-b] pyrimido [4,5-b] quinolone.

ethyl alcohol and catalytic amount of TEA afforded new compounds $4 \mathrm{a}-\mathrm{e}, 5 \mathrm{a}-\mathrm{f}, 6 \mathrm{a}-6 \mathrm{~d}, 7 \mathrm{a}-7 \mathrm{~d}$, and Scheme 3.

\section{Experimental Section}

Melting points were determined by an open capillary method and are uncorrected. The chemicals and solvents were used for laboratory grade and were purified. IR spectra were recorded (in $\mathrm{KBr}$ pallets) on Shimadzu spectrophotometer.
${ }^{1}$ HNMR spectra were recorded (in DMSO- $\mathrm{d}_{6}$ ) on Avance$300 \mathrm{MHz}$ spectrometer using TMS as an internal standard. The mass were recorded on EI-Shimadzu GC-MS spectrometer. Elemental analyses were performed on a Heraeus $\mathrm{CHN}-\mathrm{O}$ rapid analyzer.

3-Cyano-4-imino-9-methoxy-2-methythio-4H-pyrimido [2,1-b] pyrimido [4,5-b] quinoline(3). A mixture of 2-amino-7methoxy pyrimido [4,5-b] quinoline $1(2.26 \mathrm{~g}, 0.01 \mathrm{mmol})$ and bis(methylthio)methylene malononitrile 2 (1.70g, $0.01 \mathrm{mmol}$ ) was refluxed in the presence of ethyl alcohol 


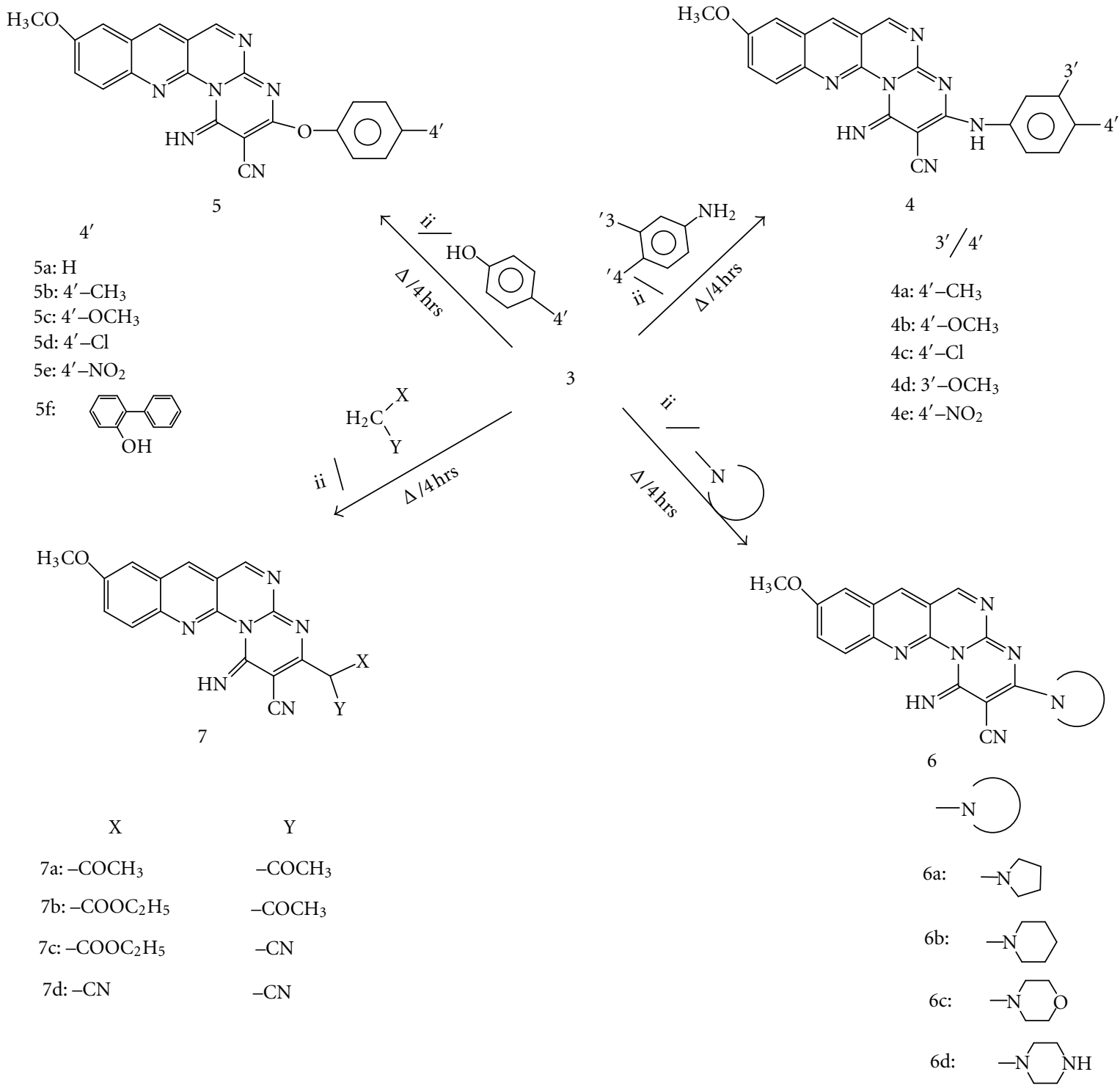

SCHEME 3

and TEA for $4 \mathrm{hr}$. The reaction mixture was cooled to room temperature and poured into ice cold water. The separated solid was filtered, washed with water, and recrystallized from $\mathrm{N}, \mathrm{N}^{\prime}$-dimethyl formamide-ethanol mixture to afford compound 3.

Brown powder, (76\% yield), Mp: $238-240^{\circ} \mathrm{C}$; EI-MS (m/z-RA\%): $372\left(\mathrm{M}^{+} \mathrm{Na}\right)$, IR $\left(\mathrm{cm}^{-1}, \mathrm{KBr}\right): 3224(=\mathrm{NH}), 2213$ (CN), ${ }^{1} \mathrm{HNMR}$ (DMSO-d 6 , ppm): $2.6\left(\mathrm{~s}, 3 \mathrm{H}, \mathrm{SCH}_{3}\right), 3.7$ (s, $\left.3 \mathrm{H}, \mathrm{Ar}-\mathrm{OCH}_{3}\right), 6.8-7.8(\mathrm{~m}, 4 \mathrm{H}, \mathrm{Ar}-\mathrm{H}),, 8.4(\mathrm{~s}, 1 \mathrm{H}-\mathrm{C}=\mathrm{CH}$ quin.) $), 8.6(\mathrm{~s}, 1 \mathrm{H},=\mathrm{NH}),{ }^{13} \mathrm{CNMR}\left(300 \mathrm{MHz}, \mathrm{DMSO}-\mathrm{d}_{6}\right.$, ppm) $15,55,80,104,115,116,121,123,125,127,135,145$, $152,163,163.8,164,165$. Anal. Calcd. For: $\mathrm{C}_{17} \mathrm{H}_{12} \mathrm{~N}_{6} \mathrm{OS}$; C, 58.61; H, 3.47; N, 24.12; Found: C, 58.50; H, 3.31; N, 24.02. 3-Cyano-4-imino-9-methoxy-2-(4'-methylanilino/4' -methoxy anilino $/ 4^{\prime}$-chloro anilino $/ 3^{\prime}$ methoxy anilino $/ 4^{\prime}$-nitro anilino)$4 H$-pyrimido $[2,1-b]$ pyrimido $[4,5-b]$ quinoline $(4 a-e)$. A mixture of compound $3(3.48 \mathrm{~g}, 0.01 \mathrm{mmol})$, ethyl alcohol catalytic amount TEA, and appropriate substituted anilines $(0.01 \mathrm{mmol})$ was refluxed for $4 \mathrm{hr}$. The reaction mixture was cooled to room temperature and poured into ice cold water. The products $4 \mathrm{a}-\mathrm{e}$ thus obtained were recrystallized from $\mathrm{N}, \mathrm{N}^{\prime}$-dimethyl formamide-ethanol solvent.

3-Cyano-4-imino-9-methoxy-2-(4'-methyl anilino)-4H-pyrimido [2,1-b] pyrimido [4,5-b] quinoline(4a). Brown powder, (85\% yield), Mp: $214^{\circ} \mathrm{C}$; IR ( $\left.\mathrm{cm}^{-1}, \mathrm{KBr}\right): 3315(\mathrm{NH}), 3222$ (=NH), $2210(\mathrm{CN}) ;{ }^{1} \mathrm{HNMR}$ (DMSO-d $\left.\mathrm{D}_{6}, \mathrm{ppm}\right)$ ) ), 2.6 (s, 3H, $\left.\mathrm{Ar}-\mathrm{CH}_{3}\right), 3.8\left(\mathrm{~s}, 3 \mathrm{H}, \mathrm{Ar}-\mathrm{OCH}_{3}\right), 4.3(\mathrm{~s}, 1 \mathrm{H},-\mathrm{NH}), 6.5-8.0$ (m, 7H, Ar-H,), 8.3 (s, 1H, CH=N-), 8.5 (s, 1H, $-\mathrm{C}=\mathrm{CH}$, ), 8.8 (s, 1H, =NH) EI-MS (m/z-RA\%): $407\left(\mathrm{M}^{+}\right)$Anal. Calcd. For: $\mathrm{C}_{23} \mathrm{H}_{17} \mathrm{~N}_{7} \mathrm{O}$; C, 67.80; $\mathrm{H}, 4.21$; N, 24.06; Found: C, $67.59 ; \mathrm{H}, 4.10 ; \mathrm{N}, 23.85$.

3-Cyano-4-imino-9-methoxy-2-(4'-methoxy anilino)-4H-pyrimido [2,1-b] pyrimido [4,5-b] quinoline(4b). Brown powder, (89\% yield), Mp: $208^{\circ} \mathrm{C}$; IR ( $\left.\mathrm{cm}^{-1}, \mathrm{KBr}\right): 3321(\mathrm{NH}), 3222$ 
(=NH), $2211(\mathrm{CN}),{ }^{1} \mathrm{HNMR}$ (DMSO-d $\left.{ }_{6}, \mathrm{ppm}\right): 3.7(\mathrm{~s}, 6 \mathrm{H}$, $\left.\mathrm{Ar}-\mathrm{OCH}_{3}\right), 4.2(\mathrm{~s}, 1 \mathrm{H},-\mathrm{NH}), 6.5-8.0(\mathrm{~m}, 7 \mathrm{H}, \mathrm{Ar}-\mathrm{H}), 8.2$ (s, $1 \mathrm{H}, \mathrm{CH}=\mathrm{N}), 8.5(\mathrm{~s}, 1 \mathrm{H},-\mathrm{C}=\mathrm{CH}), 8.6(\mathrm{~s}, 1 \mathrm{H},=\mathrm{NH})$. EIMS (m/z-RA\%): $423\left(\mathrm{M}^{+}\right)$Anal. Calcd. For: $\mathrm{C}_{23} \mathrm{H}_{17} \mathrm{~N}_{7} \mathrm{O}_{2} ; \mathrm{C}$, 65.24; H, 4.05; N, 23.16; Found: C, 65.02; H, 3.82; N, 23.00. 3-Cyano-4-imino-9-methoxy-2-(4'-chloro anilino)-4H-pyrimido [2,1-b] pyrimido [4,5-b] quinoline(4c). Brown powder, (80\% yield), Mp: $219^{\circ} \mathrm{C}$; IR $\left(\mathrm{cm}^{-1}, \mathrm{KBr}\right): 3330(\mathrm{NH}), 3224$ $(=\mathrm{NH}) ; 2210(\mathrm{CN}),{ }^{1} \mathrm{HNMR}\left(\mathrm{DMSO}-\mathrm{d}_{6}, \mathrm{ppm}\right): 3.7$ (s, 3H, $\left.\mathrm{Ar}-\mathrm{OCH}_{3}\right), 4.0(\mathrm{~s}, 1 \mathrm{H},-\mathrm{NH}), 6.4-8.0(\mathrm{~m}, 7 \mathrm{H}, \mathrm{Ar}-\mathrm{H}), 8.1(\mathrm{~s}$, $1 \mathrm{H}, \mathrm{CH}=\mathrm{N}-), 8.5(\mathrm{~s}, 1 \mathrm{H},-\mathrm{C}=\mathrm{CH}), 8.6(\mathrm{~s}, 1 \mathrm{H},=\mathrm{NH})$. EI-MS (m/z-RA\%): $428(\mathrm{M}+)$ Anal. Calcd. For: $\mathrm{C}_{22} \mathrm{H}_{14} \mathrm{Cl} \mathrm{N} \mathrm{N}_{7} \mathrm{O}$; C, 61.76; H, 3.30; N, 22.92; Found: C, 61.05; H, 2.56; N, 22.28.

3-Cyano-4-imino-9-methoxy-2-(3'-methoxy anilino)-4H-pyrimido [2,1-b] pyrimido [4,5-b] quinoline(4d). Brown powder, (66\% yield), Mp: $217^{\circ} \mathrm{C}$; IR $\left(\mathrm{cm}^{-1}, \mathrm{KBr}\right): 3331(\mathrm{NH}), 3220$ $(=\mathrm{NH}), 2208(\mathrm{CN}) ;{ }^{1} \mathrm{HNMR}\left(\mathrm{DMSO}_{-} \mathrm{d}_{6}, \mathrm{ppm}\right): 3.8(\mathrm{~s}, 6 \mathrm{H}$, $\left.-\mathrm{OCH}_{3}\right), 4.0(\mathrm{~s}, 1 \mathrm{H},-\mathrm{NH}), 6.4-8.0(\mathrm{~m}, 7 \mathrm{H}, \mathrm{Ar}-\mathrm{H}), 8.1(\mathrm{~s}, 1 \mathrm{H}$, $\mathrm{CH}=\mathrm{N}-), 8.5(\mathrm{~s}, 1 \mathrm{H},-\mathrm{C}=\mathrm{CH}) 8.6(\mathrm{~s}, 1 \mathrm{H},=\mathrm{NH})$. EI-MS $\left(\mathrm{m} / \mathrm{z}^{-}\right.$ RA\%): $423(\mathrm{M}+)$ Anal. Calcd. For: $\mathrm{C}_{23} \mathrm{H}_{17} \mathrm{~N}_{7} \mathrm{O}_{2} ; \mathrm{C}, 65.24 ; \mathrm{H}$, 4.05; N, 23.16; Found: C, 64.70; H, 3.64; N, 22.62.

3-Cyano-4-imino-9-methoxy-2-(4'-nitro anilino)-4H-pyrimido [2,1-b] pyrimido [4,5-b] quinoline(4e). Brown powder, (62\% yield), Mp: $213^{\circ} \mathrm{C}$; IR $\left(\mathrm{cm}^{-1}, \mathrm{KBr}\right): 3318(\mathrm{NH}), 3219$ $(=\mathrm{NH}), 2210(\mathrm{CN}) ;{ }^{1} \mathrm{HNMR}\left(\mathrm{DMSO}-\mathrm{d}_{6}, \mathrm{ppm}\right): 2.6(\mathrm{~s}, 3 \mathrm{H}$, $\left.\mathrm{Ar}-\mathrm{CH}_{3}\right), 3.8\left(\mathrm{~s}, 3 \mathrm{H},-\mathrm{OCH}_{3}\right), 4.0(\mathrm{~s}, 1 \mathrm{H},-\mathrm{NH}), 6.4-8.0(\mathrm{~m}$, $7 \mathrm{H}, \mathrm{Ar}-\mathrm{H}), 8.0(\mathrm{~s}, 1 \mathrm{H}, \mathrm{CH}=\mathrm{N}-), 8.5(\mathrm{~s}, 1 \mathrm{H},-\mathrm{C}=\mathrm{CH}), 8.6(\mathrm{~s}$, $1 \mathrm{H},=\mathrm{NH})$. EI-MS (m/z-RA\%): $438\left(\mathrm{M}^{+}\right)$Anal. Calcd. For: $\mathrm{C}_{22} \mathrm{H}_{14} \mathrm{~N}_{8} \mathrm{O}_{3}$; C, 60.27; H, 3.22; N, 25.56; Found: C, 59.67; H, $2.72 ; \mathrm{N}, 24.84$.

3-Cyano-4-imino-9-methoxy-2-(phenoxy/4'-methyl phenoxy/ $4^{\prime}$-methoxy phonoxy $/ 4^{\prime}$-chloro phenoxy $/ 4^{\prime}$-nitro phonoxy $/ 2^{\prime}$ hydroxy biphenoxy)-4H-pyrimido [2,1-b] pyrimido [4,5b] quinoline $(5 a-f)$. A mixture of compound 3 (3.48g, $0.01 \mathrm{mmol})$, ethyl alcohol catalytic amount of triethyl amine, and appropriate substituted phenols $(0.01 \mathrm{mmol})$ was refluxed for $4 \mathrm{hrs}$. The reaction mixture was cooled to room temperature and poured into ice cold water. The products $5 a-f$ thus obtained were recrystallized from $\mathrm{N}, \mathrm{N}^{\prime}$-dimethyl formamide-ethanol solvent.

3-Cyano-4-imino-9-methoxy-2-(phenoxy)-4H-pyrimido [2,1b] pyrimido [4,5-b] quinoline (5a). Brown powder, (82\% yield), Mp: $204-205^{\circ} \mathrm{C}$, IR ( $\left.\mathrm{cm}^{-1}, \mathrm{KBr}\right): 3222(=\mathrm{NH}), 2209$ (CN); ${ }^{1} \mathrm{HNMR}$ (DMSO-d 6 , ppm): 3.5 (s, 3H, $\mathrm{Ar}-\mathrm{OCH}_{3}$ ), 6.6-8.5 (m, 9H, Ar-H), $8.4(\mathrm{~s}, 1 \mathrm{H},=\mathrm{NH}), 8.5(\mathrm{~s}, 1 \mathrm{H}, \mathrm{CH}=\mathrm{C}$ quin.); EI-MS (m/z-RA\%): $395\left(\mathrm{M}^{+} 1\right)$ Anal. Calcd. For: $\mathrm{C}_{22} \mathrm{H}_{14} \mathrm{~N}_{6} \mathrm{O}_{2}$; C, 67.00; H, 3.58; N, 21. 31, Found: C, 66.49; $\mathrm{H}, 3.01 ; \mathrm{N}, 21.01$.

3-Cyano-4-imino-9-methoxy-2-(4'-methyl phenoxy)-4H-pyrimido [2,1-b] pyrimido [4,5-b] quinoline(5b). Brown powder, (78\% yield), Mp: $202-204^{\circ} \mathrm{C}$; IR ( $\left.\mathrm{cm}^{-1}, \mathrm{KBr}\right): 3218$ $(=\mathrm{NH}), 2213(\mathrm{CN}) ;{ }^{1} \mathrm{HNMR}$ (DMSO-d $\left.\mathrm{d}_{6}, \mathrm{ppm}\right): 3.8(\mathrm{~s}, 3 \mathrm{H}$, $\left.\mathrm{Ar}-\mathrm{OCH}_{3}\right), 6.6-7.9(\mathrm{~m}, 8 \mathrm{H}, \mathrm{Ar}-\mathrm{H}), 8.5$ (s, $1 \mathrm{H}, \mathrm{CH}=\mathrm{C}$ quin.); $8.6(\mathrm{~s}, 1 \mathrm{H},=\mathrm{NH})$. EI-MS (m/z-RA\%): $408\left(\mathrm{M}^{+}\right)$Anal. Calcd. For: $\mathrm{C}_{23} \mathrm{H}_{16} \mathrm{~N}_{6} \mathrm{O}_{2} ; \mathrm{C}, 67.64 ; \mathrm{H}, 3.95 ; \mathrm{N}, 20.58$ Found: $\mathrm{C}, 67.02$; $\mathrm{H}, 3.14 ; \mathrm{N}, 19.85$.
3-Cyano-4-imino-9-methoxy-2-(4'-methoxy phonoxy)-4Hpyrimido [2,1-b] pyrimido [4,5-b] quinoline(5c). Brown powder, (74\% yield). Mp: $207^{\circ} \mathrm{C}$; IR $\left(\mathrm{cm}^{-1}, \mathrm{KBr}\right): 3220$ (=NH), $2209(\mathrm{CN}) ;{ }^{1} \mathrm{HNMR}$ (DMSO-d $\left.{ }_{6}, \mathrm{ppm}\right): 3.6$ (s, 3H, $\left.\mathrm{Ar}-\mathrm{OCH}_{3}\right), 6.6-7.8(\mathrm{~m}, 8 \mathrm{H}, \mathrm{Ar}-\mathrm{H}), 8.3(\mathrm{~s}, 1 \mathrm{H}, \mathrm{CH}=\mathrm{C}$ quin.), $8.5(\mathrm{~s}, 1 \mathrm{H},=\mathrm{NH})$. EI-MS (m/z-RA\%): $424\left(\mathrm{M}^{+}\right)$Anal. Calcd. For: $\mathrm{C}_{23} \mathrm{H}_{16} \mathrm{~N}_{6} \mathrm{O}_{3}$, Calcd: C, 65.09; H, 3.80; N, 19.80, Found: C, 64.48; H, 2.60; N, 19.18 .

3-Cyano-4-imino-9-methoxy-2-(4'-chloro phenoxy)-4H-pyrimido [2,1-b] pyrimido [4,5-b] quinoline(5d). Brown powder, (70\% yield), Mp: $214-216^{\circ} \mathrm{C}$; IR $\left(\mathrm{cm}^{-1}, \mathrm{KBr}\right): 3219$ $(=\mathrm{NH}), 2213(\mathrm{CN}) ;{ }^{1} \mathrm{HNMR}$ (DMSO-d $\left.{ }_{6}, \mathrm{ppm}\right): 3.7(\mathrm{~s}, 3 \mathrm{H}$, Ar- $\left.\mathrm{OCH}_{3}\right), 6.6-7.9(\mathrm{~m}, 8 \mathrm{H}, \mathrm{Ar}-\mathrm{H}), 8.3(\mathrm{~s}, 1 \mathrm{H}, \mathrm{CH}=\mathrm{C}$ quin.), $8.5(\mathrm{~s}, 1 \mathrm{H},=\mathrm{NH})$. EI-MS (m/z-RA\%): $429\left(\mathrm{M}^{+} 1\right)$ Anal. Calcd. For: $\mathrm{C}_{22} \mathrm{H}_{13} \mathrm{ClN}_{6} \mathrm{O}_{2} ; \mathrm{C}, 61.62 ; \mathrm{H}, 3.06 ; \mathrm{N}, 19.60$. Found: $\mathrm{C}$, 61.02; H, 2.70; N, 19.13.

3-Cyano-4-imino-9-methoxy-2-(4'-nitro phonoxy)-4H-pyrimido [2,1-b] pyrimido [4,5-b] quinoline(5e). Brown powder, (73\% yield), Mp: $211-213^{\circ} \mathrm{C}$; IR $\left(\mathrm{cm}^{-1}, \mathrm{KBr}\right): 3222$ (=NH), 2209 (CN); ${ }^{1} \mathrm{HNMR}$ (DMSO-d $\left.{ }_{6}, \mathrm{ppm}\right): 3.5$ (s, 3H, Ar- $\left.\mathrm{OCH}_{3}\right), 6.7-8.0(\mathrm{~m}, 8 \mathrm{H}, \mathrm{Ar}-\mathrm{H}), 8.5(\mathrm{~s}, 1 \mathrm{H}, \mathrm{CH}=\mathrm{C}$ quin.) $8.7(\mathrm{~s}, 1 \mathrm{H},=\mathrm{NH})$. EI-MS (m/z-RA\%): $439\left(\mathrm{M}^{+}\right)$Anal. Calcd. For: $\mathrm{C}_{22} \mathrm{H}_{13} \mathrm{~N}_{7} \mathrm{O}_{4} ; \mathrm{C}, 60.14 ; \mathrm{H}, 2.98 ; \mathrm{N}, 22.31$, Found: C, $60.00 ; \mathrm{H}, 2.60 ; \mathrm{N}, 22.02$.

3-Cyano-4-imino-9-methoxy-2-(2'-hydroxy biphenoxy)-4Hpyrimido $[2,1-b]$ pyrimido [4,5-b] quinoline(5f). Brown powder, (82\% yield), Mp: $224-226^{\circ} \mathrm{C}$; IR ( $\left.\mathrm{cm}^{-1}, \mathrm{KBr}\right): 3220$ (=NH); 2209 (CN), ${ }^{1} \mathrm{HNMR}$ (DMSO-d $\left.\mathrm{d}_{6}, \mathrm{ppm}\right): 3.7$ (s, 3H, $\left.\mathrm{Ar}-\mathrm{OCH}_{3}\right), 6.7-7.9(\mathrm{~m}, 13 \mathrm{H}, \mathrm{Ar}-\mathrm{H}), 8.5(\mathrm{~s}, 1 \mathrm{H}, \mathrm{CH}=\mathrm{C}$ quin.), $8.6(\mathrm{~s}, 1 \mathrm{H},=\mathrm{NH})$. EI-MS (m/z-RA\%): $470\left(\mathrm{M}^{+}\right)$Anal. Calcd. For: $\mathrm{C}_{28} \mathrm{H}_{18} \mathrm{~N}_{6} \mathrm{O}_{2}$; Calcd: C, 71.48; H, 3.86; N, 17.66; Found: C, 71.00; H, 3.41; N,17.43.

3-Cyano-4-imino-9-methoxy-2-(pyrrolidino/piperidino/morpholino/piperazino)-4H-pyrimido $[2,1-b]$ pyrimido $[4,5-$ b] quinolone $(6 a-d)$. A mixture of compound $3(3.48 \mathrm{~g}$, $0.01 \mathrm{mmol}$ ), ethyl alcohol catalytic amount of triethyl amine, and appropriate cyclic secondary amines $(0.01 \mathrm{mmol})$ was refluxed for $4 \mathrm{hrs}$ and the mixture was poured into ice cold water. The products $6 \mathrm{a}-\mathrm{d}$ thus obtained were recrystallized from $\mathrm{N}, \mathrm{N}^{\prime}$-dimethyl formamide-ethanol solvent.

3-Cyano-4-imino-9-methoxy-2-(pyrrolidino)-4H-pyrimido [2,1-b] pyrimido [4,5-b] quinoline (6a). Brown powder, (82\% yield), Mp: $218^{\circ} \mathrm{C}$; IR $\left(\mathrm{cm}^{-1}, \mathrm{KBr}\right): 3216(=\mathrm{NH}) ; 2211(\mathrm{CN})$, ${ }^{1} \mathrm{HNMR}$ (DMSO-d $\left.\mathrm{d}_{6}, \mathrm{ppm}\right): 1.6\left(\mathrm{t}, 4 \mathrm{H}, \mathrm{CH}_{2}\right), 2.7(\mathrm{t}, 4 \mathrm{H}$, $-\mathrm{NCH}_{2}-$ ), 3.7 (s, 3H, Ar- $\left.\mathrm{OCH}_{3}\right), 6.7-7.8(\mathrm{~m}, 4 \mathrm{H}, \mathrm{Ar}-\mathrm{H}), 8.5$ (s, $1 \mathrm{H}, \mathrm{CH}=\mathrm{C}$ quin.). $8.8(\mathrm{~s}, 1 \mathrm{H},=\mathrm{NH})$. EI-MS $(\mathrm{m} / \mathrm{z}-\mathrm{RA} \%)$ : $371\left(\mathrm{M}^{+}\right)$Anal. Calcd. For: $\mathrm{C}_{20} \mathrm{H}_{17} \mathrm{~N}_{7} \mathrm{O} ; \mathrm{C}, 64.68 ; \mathrm{H}, 4.61 ; \mathrm{N}$, 26.40; Found: C, 64.12; H, 4.10; N, 25.92.

3-Cyano-4-imino-9-methoxy-2-(piperidino)-4H-pyrimido [2,1-b] pyrimido [4,5-b] quinoline (6b). Brown powder, $(80 \%$ yield), Mp: $222-224^{\circ} \mathrm{C}$; IR ( $\left.\mathrm{cm}^{-1}, \mathrm{KBr}\right): 3216(=\mathrm{NH}) ; 2208$ (CN), ${ }^{1} \mathrm{HNMR}$ (DMSO- $\left.\mathrm{d}_{6}, \mathrm{ppm}\right): 1.5\left(\mathrm{t}, 6 \mathrm{H}\right.$, three $-\mathrm{CH}_{2}-$ ), $2.8\left(\mathrm{t}, 4 \mathrm{H}\right.$, two- $\left.\mathrm{NCH}_{2}-\right), 3.7\left(\mathrm{~s}, 3 \mathrm{H}, \mathrm{Ar}-\mathrm{OCH}_{3}\right), 6.7-7.8(\mathrm{~m}$, $4 \mathrm{H}, \mathrm{Ar}-\mathrm{H}), 8.5(\mathrm{~s}, 1 \mathrm{H}, \mathrm{CH}=\mathrm{C}$ quin.); $8.6(\mathrm{~s}, 1 \mathrm{H},=\mathrm{NH})$. EI-MS (m/z-RA\%): $385\left(\mathrm{M}^{+}\right)$; Anal. Calcd. For: $\mathrm{C}_{21} \mathrm{H}_{19} \mathrm{~N}_{7} \mathrm{O}$; C, 65.44; H, 4.97; N, 25.44, Found: C, 65.15, H, 4.20; N, 25.02 . 
3-Cyano-4-imino-9-methoxy-2-(morpholino)-4H-pyrimido [2,1-b] pyrimido [4,5-b] quinolne (6c). Brown powder, (88\% yield), Mp: $228-230^{\circ} \mathrm{C}$; IR $\left(\mathrm{cm}^{-1}, \mathrm{KBr}\right): 3222(=\mathrm{NH}) ; 2211$ (CN), ${ }^{1}$ HNMR (DMSO-d $\left.\mathrm{d}_{6}, \mathrm{ppm}\right): 2.7\left(\mathrm{t}, 4 \mathrm{H},-\mathrm{NCH}_{2}\right), 3.6$ (s, $\left.3 \mathrm{H}, \mathrm{Ar}-\mathrm{OCH}_{3}\right), 3.8\left(\mathrm{t}, 4 \mathrm{H}, \mathrm{OCH}_{2}\right), 6.9-7.9(\mathrm{~m}, 4 \mathrm{H}$, Ar-H), 8.5 (s, $1 \mathrm{H}, \mathrm{CH}=\mathrm{C}$ quin.). 8.7(s, $1 \mathrm{H},=\mathrm{NH})$. EI-MS (m/z-RA\%): $388(\mathrm{M}+1)$ Anal. Calcd. For: $\mathrm{C}_{20} \mathrm{H}_{17} \mathrm{~N}_{7} \mathrm{O}_{2}$. Calcd: C, 62.01; H, 4.42; N, 25.31; Found: C, 61.60; H, 4.00; $\mathrm{N}, 24.82$.

3-Cyano-4-imino-9-methoxy-2-(piperazino)-4H-pyrimido [2,1-b] pyrimido [4,5-b] quinolone (6d). Brown powder, (85\% yield), Mp: $212-214^{\circ} \mathrm{C}$; IR $\left(\mathrm{cm}^{-1}, \mathrm{KBr}\right): 3226(=\mathrm{NH})$; 2210(CN), ${ }^{1} \mathrm{HNMR}$ (DMSO-d $\left.\mathrm{d}_{6}, \mathrm{ppm}\right): 2.1$ (s, 1H, NH), 2.7 $\left(\mathrm{m}, 8 \mathrm{H}\right.$, four $\left.\mathrm{CH}_{2}, \mathrm{~J}=7.5 \mathrm{~Hz}\right), 3.4\left(\mathrm{~s}, 3 \mathrm{H}, \mathrm{Ar}-\mathrm{OCH}_{3}\right), 6.7-7.9$ $(\mathrm{m}, 4 \mathrm{H}, \mathrm{Ar}-\mathrm{H}), 8.5$ (s, $1 \mathrm{H}, \mathrm{CH}=\mathrm{C}$ quin.), $8.6(\mathrm{~s}, 1 \mathrm{H},=\mathrm{NH})$. EI-MS (m/z-RA\%): $387(\mathrm{M}+1)$ Anal. Calcd. For: $\mathrm{C}_{20} \mathrm{H}_{18} \mathrm{~N}_{8} \mathrm{O}$; C, 62.17; H, 4.70; N, 29.00; Found: C, 61.81; H, 4.34; N, 28.52. 3-Cyano-4-imino-9-methoxy-2-( $\alpha$-acetyl acetonyl/ $\alpha$-ethylacetoacetyl/ $\alpha$-ethylcyanoacetyl/ $\alpha$-malononitriyl)- $4 H$-pyrimido [2,1-b] pyrimido [4,5-b] quinoline (7a-d). A mixture of compound 3 (3.48 g, $0.01 \mathrm{mmol}$ ), ethyl alcohol catalytic amount of triethyl amine, and appropriate compounds containing an active methylene group $(0.01 \mathrm{mmol})$ was refluxed for $4 \mathrm{hrs}$. The reaction mixture was cooled at room temperature and poured into ice cold water. The products $7 \mathrm{a}-\mathrm{d}$ thus obtained were recrystallized from $\mathrm{N}, \mathrm{N}^{\prime}$-dimethyl formamide-ethanol. 3-Cyano-4-imino-9-methoxy-2-( $\alpha$-acetyl acetonyl)-4H-pyrimido [2,1-b] pyrimido [4,5-b] quinolone (7a). Brown powder, (75\% yield), Mp: $205-207^{\circ} \mathrm{C}$; IR $\left(\mathrm{cm}^{-1}, \mathrm{KBr}\right): 3322(=\mathrm{NH})$; $2210(\mathrm{CN}), 1685$ (CO), ${ }^{1} \mathrm{HNMR}$ (DMSO-d $\left.\mathrm{d}_{6}, \mathrm{ppm}\right): 2.2$ (s, $6 \mathrm{H}$, two $\left.\mathrm{COCH}_{3}\right), 3.6\left(\mathrm{~s}, 3 \mathrm{H}, \mathrm{Ar}-\mathrm{OCH}_{3}\right), 3.9(\mathrm{~s}, 1 \mathrm{H},-\mathrm{CH})$, 6.8-7.7 (m, $4 \mathrm{H}, \mathrm{Ar}-\mathrm{H}$ ), 8.5 (s, $1 \mathrm{H}, \mathrm{CH}=\mathrm{C}$ quin.); 8.8 (s, $1 \mathrm{H},=\mathrm{NH})$; EI-MS (m/z-RA\%): 401(M+1) Anal. Calcd. For: $\mathrm{C}_{21} \mathrm{H}_{16} \mathrm{~N}_{6} \mathrm{O}_{3}$; C, 62.99; H, 4.03; N, 20.99; Found: C, 62.35; H, 2.64; N, 20.42.

3-Cyano-4-imino-9-methoxy-2-( $\alpha$-ethylacetoacetyl)-4H-pyrimido [2,1-b ]pyrimido [4,5-b] quinolone (7b). Brown powder, (77\% yield), Mp: $212^{\circ} \mathrm{C}$; IR ( $\left.\mathrm{cm}^{-1}, \mathrm{KBr}\right): 3400(=\mathrm{NH}) ; 2209$ (CN), 1733 (CO of ester), 1684 (CO), 1245 (C-O); ${ }^{1}$ HNMR (DMSO- $\left.\mathrm{d}_{6}, \mathrm{ppm}\right): 1.4\left(\mathrm{~s}, 3 \mathrm{H},-\mathrm{CH}_{3}\right), 2.2\left(\mathrm{~s}, 3 \mathrm{H}, \mathrm{COCH}_{3}\right), 3.7$ (s, $\left.3 \mathrm{H}, \mathrm{Ar}-\mathrm{OCH}_{3}\right), 3.9(\mathrm{~s}, 1 \mathrm{H},-\mathrm{CH}), 4.2\left(\mathrm{~d}, 2 \mathrm{H},-\mathrm{OCH}_{2}-\right)$, 6.8-7.9 (m, $4 \mathrm{H}, \mathrm{Ar}-\mathrm{H}), 8.6$ (s, $1 \mathrm{H}, \mathrm{CH}=\mathrm{C}$ quin.); $8.8(\mathrm{~s}, 1 \mathrm{H}$, $=\mathrm{NH}$ ); Anal. Calcd. For: $\mathrm{C}_{22} \mathrm{H}_{18} \mathrm{~N}_{6} \mathrm{O}_{4} ; \mathrm{C}, 61.39 ; \mathrm{H}, 4.22 ; \mathrm{N}$, 19.53; Found: C, 60.84; H, 3.92; N, 19.02.

3-Cyano-4-imino-9-methoxy-2-( $\alpha$-ethylcyanoacetyl)-4H-pyrimido [2,1-b] pyrimido [4,5-b] quinolone (7c). Brown powder, (84\% yield), m.p. $218-220^{\circ} \mathrm{C}$; IR $\left(\mathrm{cm}^{-1}, \mathrm{KBr}\right): 3338(=\mathrm{NH})$; 2224 (CN), 1740 (CO of ester), 1258 (C-O); ${ }^{1} \mathrm{HNMR}$ (DMSO-d $\left.\mathrm{d}_{6}, \mathrm{ppm}\right): 1.4\left(\mathrm{~s}, 3 \mathrm{H},-\mathrm{CH}_{3}\right), 3.7\left(\mathrm{~s}, 3 \mathrm{H}, \mathrm{Ar}-\mathrm{OCH}_{3}\right)$, $3.9(\mathrm{~s}, 1 \mathrm{H},-\mathrm{CH}), 4.2\left(\mathrm{~s}, 2 \mathrm{H},-\mathrm{OCH}_{2}-\right), 6.9-7.8(\mathrm{~m}, 4 \mathrm{H}$, $\mathrm{Ar}-\mathrm{H}), 8.7$ (s, 1H, CH=C quin.); $8.9(\mathrm{~s}, 1 \mathrm{H},=\mathrm{NH})$; EI-MS (m/z-RA\%): $413\left(\mathrm{M}+\right.$ ) Anal. Calcd. For: $\mathrm{C}_{21} \mathrm{H}_{15} \mathrm{~N}_{7} \mathrm{O}_{3}$; C, 61.01; H, 3.66; N, 23.72. Found: C, 60.50; H, 3.05; N, 23.28. 3-Cyano-4-imino-9-methoxy-2-( $\alpha$-malanonitriyl)-4H-pyrimido [2,1-b] pyrimido [4,5-b] quinolone (7d). Brown powder, (79\% yield), $\mathrm{Mp}: 226-228^{\circ} \mathrm{C}$; IR $\left(\mathrm{cm}^{-1}, \mathrm{KBr}\right): 3418$ $(=\mathrm{NH}) ; 2211(\mathrm{CN}),{ }^{1} \mathrm{HNMR}$ (DMSO-d $\left.\mathrm{d}_{6}, \mathrm{ppm}\right): 3.7$ (s,3H,
$\left.\mathrm{Ar}-\mathrm{OCH}_{3}\right), 4.3(\mathrm{~s}, 1 \mathrm{H},-\mathrm{CH}), 6.6-7.8(\mathrm{~m}, 4 \mathrm{H}, \mathrm{Ar}-\mathrm{H}), 8.5$ (s, $1 \mathrm{H}, \mathrm{CH}=\mathrm{C}$ quin.); $8.8(\mathrm{~s}, 1 \mathrm{H},=\mathrm{NH}) ; \mathrm{EI}-\mathrm{MS}(\mathrm{m} / \mathrm{z}-\mathrm{RA} \%)$ : 367(M+1); Anal. Calcd. For: $\mathrm{C}_{19} \mathrm{H}_{10} \mathrm{~N}_{8} \mathrm{O}$; C, 62.29; H, 2.75; N, 30.59; Found: C, 61.56; H, 2.18; N, 30.02.

\section{Result and Discussion}

In the present investigation, we have developed new methodology towards the synthesis of 3-cyano-4-imino-9-methoxy2-methythio- $4 \mathrm{H}$-pyrimido [2,1-b] pyrimido [4,5-b] quinoline and their substituted derivatives. 3 Our method gives single product with high yield. The reaction started with 2-amino-7-methoxypyrimido [4,5-b] quinoline $\mathbf{1}$ and bis (methylthio)methylene malononitrile $\mathbf{2}$ were refluxed in ethyl alcohol in presence of catalytic amount of triethyl amine (TEA) to afford $\mathbf{3}$, Scheme 1.

Compound 3 posses a replaceable active methylthio group at 2-position which is activated by ring 1-nitrogen atom and electron withdrawing group 3-cyano group. Compound 3 reacted with selected $\mathrm{N}-$, O-, C-nucleophiles like aryl amines hetryl amines, substituted phenols, and compounds containing an active methylene group. The compound (3) on independent reaction with p-methyl aniline, and p-methoxy aniline, $\mathrm{p}$-chloro aniline, $\mathrm{m}$-methoxy aniline, $\mathrm{p}$-nitro aniline, in ethyl alcohol and catalytic amount of triethyl amine, afforded 3-cyano-4-imino-9-methoxy-2-(4'-methyl anilino/ $4^{\prime}$-methoxy anilino $/ 4^{\prime}$-chloro anilino $/ 3^{\prime}$ methoxy anilino $/ 4^{\prime}$ nitro anilino)-4H-pyrimido [2,1-b] pyrimido [4,5-b] quinoline (4a-e), respectively, Scheme 3.

Under similar experimental condition compound 3 reacted independently with hetryl amines like pyrolidine, piperidine, morpholine, and piperazine to yield 3-cyano4-imino-9-methoxy-2-(pyrolidino/piperidino/morpholino/ piperazino)-4H-pyrimido [2,1-b] pyrimido [4,5-b] quinoline. (6a-d), respectively, Scheme 3.

3-Cyano-4-imino-9-methoxy-2-(phenoxy/4'-methyl phenoxy $/ 4^{\prime}$-methoxy phonoxy $/ 4^{\prime}$-chloro phenoxy $/ 4^{\prime}$-nitro phonoxy/2'-hydroxy biphenoxy)-4H-pyrimido $[2,1-\mathrm{b}]$ pyrimido [4,5-b] quinoline (5a-f), respectively. Scheme 3 were obtained by condensation of 3 with phenol, p-methyl phenol, p-methoxy phenol, p-chloro phenol, p-nitro phenol, ohydroxy biphenol, in ethyl alcohol and catalytic amount of TEA.

Compounds on reaction with acetyl acetone, ethyl acetoacetate, ethyl cyano acetate, malanonitrile in presence of ethyl alcohol and catalytic amount of TEA yielded compounds 3 -cyano-4-imino-9-methoxy-2-( $\alpha$-acetyl acetonyl/ $\alpha$-ethylacetoacetyl $/ \alpha$-ethylcyanoacetyl $/ \alpha$-malononitriyl)- $4 \mathrm{H}$-pyrimido [2,1-b] pyrimido [4,5-b] quinoline (7a-d), respectively, Scheme 3.

Compounds $4 a-e, 5 a-f, 6 a-6 d$, and 7a-7d show absorption bands in their IR spectra in the range of $3200 \mathrm{~cm}^{-1}$ to $3460 \mathrm{~cm}^{-1}$ and $2190 \mathrm{~cm}^{-1}$ to $2230 \mathrm{~cm}^{-1}$ due to $=\mathrm{NH}$ and $\mathrm{CN}$ stretching, respectively, ${ }^{1} \mathrm{HNMR}$ and Mass spectral data are also in agreement with structures of newly synthesized compounds $4 a-e, 5 a-f, 6 a-6 d$, and $7 a-7 d$.

The structures of these newly synthesized compounds were established on the basis of elemental analysis, IR, PMR, 
TABLE 1: Antimicrobial activity of compounds (4a-e, 5a-f, 6a-6d, and 7a-7d).

\begin{tabular}{|c|c|c|c|c|c|}
\hline \multirow{3}{*}{ Serial number } & \multirow{3}{*}{ Compound code } & \multicolumn{4}{|c|}{ Zone of inhibition in $\mathrm{mm}$} \\
\hline & & \multicolumn{2}{|c|}{ Fungal species } & \multicolumn{2}{|c|}{ Bacterial species } \\
\hline & & Aspergillus flavus & Aspergillus niger & E. coli & B. subtilis \\
\hline 1 & 3 & - & - & 16 & 22 \\
\hline 2 & $4 \mathrm{a}$ & 12 & 10 & 10 & 26 \\
\hline 3 & $4 \mathrm{~b}$ & 14 & 13 & 12 & 13 \\
\hline 4 & $4 c$ & 16 & 17 & 20 & 18 \\
\hline 5 & $4 d$ & 10 & 13 & 10 & 15 \\
\hline 6 & $4 \mathrm{e}$ & 12 & 16 & 22 & 21 \\
\hline 7 & $5 \mathrm{a}$ & 15 & 15 & 13 & 23 \\
\hline 8 & $5 b$ & 08 & 06 & 10 & 21 \\
\hline 9 & $5 c$ & 11 & 16 & 13 & 06 \\
\hline 10 & $5 d$ & 14 & 13 & 22 & 18 \\
\hline 11 & $5 e$ & 12 & 15 & 18 & 13 \\
\hline 12 & $5 f$ & 06 & 14 & 10 & 23 \\
\hline 13 & $6 a$ & 09 & 16 & 13 & 10 \\
\hline 14 & $6 b$ & 17 & 15 & 26 & 13 \\
\hline 15 & $6 c$ & 11 & 12 & 12 & 22 \\
\hline 16 & $6 \mathrm{~d}$ & 15 & 10 & 09 & 21 \\
\hline 17 & $7 \mathrm{a}$ & 13 & 13 & 04 & 16 \\
\hline 18 & $7 \mathrm{~b}$ & 05 & 15 & 11 & 13 \\
\hline 19 & $7 \mathrm{c}$ & 09 & 12 & 10 & 17 \\
\hline 20 & $7 \mathrm{~d}$ & 13 & 18 & 26 & 23 \\
\hline \multirow{2}{*}{\multicolumn{2}{|c|}{ Positive control }} & 26 & 25 & 32 & 30 \\
\hline & & \multicolumn{2}{|c|}{ Fluconazole } & \multicolumn{2}{|c|}{ Streptomycin } \\
\hline
\end{tabular}

and MASS spectral data, spectral studies of all compounds show that compounds are stable and do not exhibit any tautomerism.

\section{Antimicrobial Activity}

The synthesized compounds were evaluated for their antifungal and antibacterial activity against species Aspergillus flavus, Aspergillus niger, and E. coli and B. subtilis by paper disc diffusion method [27]. All the synthesized compounds were dissolved in Dimethyl sulphoxide. The synthesized compounds exhibited zone of inhibition of $09-23 \mathrm{~mm}$ in diameter where as standard Fluconazole exhibited zone of inhibition of 26 and $25 \mathrm{~mm}$ Streptomycin exhibited zone of inhibition of 32 and $30 \mathrm{~mm}$ in diameter against $E$. coli and B. subtilis, respectively. Amongst the synthesized compounds 3, compound $4 \mathrm{c}(16,17 \mathrm{~mm}), 4 \mathrm{e}(12,16 \mathrm{~mm}), 5 \mathrm{~d}(14$, $13 \mathrm{~mm}), 5 \mathrm{e}(12,15 \mathrm{~mm}), 6 \mathrm{~b}(17,15 \mathrm{~mm})$, and $7 \mathrm{~d}(13,18 \mathrm{~mm})$ showed higher zone of inhibition against Aspergillus flavus, Aspergillus niger, respectively. And 4c (20, $18 \mathrm{~mm}), 4 \mathrm{e}(22$, $21 \mathrm{~mm}), 5 \mathrm{~d}(22,18 \mathrm{~mm}), 5 \mathrm{e}(18,13 \mathrm{~mm}), 6 \mathrm{~b}(26,13 \mathrm{~mm})$, and $7 \mathrm{~d}(26,23 \mathrm{~mm})$ showed higher zone of inhibition against E. coli and B. subtilis. It seems that the presence of nitro and chloro group increases antifungal activity (Table 1).

\section{Conclusion}

In this communication all synthesized compounds reported first time and describe the simple route of their synthesis in mild condition with good yield. The present study showed that all the title compounds were exhibiting significant antibacterial and antifungal activities. However, further studies are required to establish the mechanism of action of the title compounds. From the screening data, it was found that $4 \mathrm{c}, 4 \mathrm{e}, 5 \mathrm{~d}, 5 \mathrm{e}, 6 \mathrm{~b}$, and $7 \mathrm{~d}$ derivative have encouraging antibacterial and antifungal activity, which needs to be further investigated to get better antibacterial and antifungal agents.

\section{Acknowledgment}

The authors are grateful to Dr. N. V. Kalyankar, Principal, Yeshwant Mahavidyalaya, Nanded, for providing laboratory facilities, to UGC New Delhi for financial assistance under major research project (F.N. 39-834/2010 (SR)), Director, IICT, Hyderabad, for providing spectra, and to the Principal, Dr Kalamse, N.E.S Science College Nanded, for biological activity. 


\section{References}

[1] R. R. Tumer, R. R. Woodward, and R. H. F. Manske, Eds., vol. 3, chapter 3, Academic Press, New York, NY, USA, 1953.

[2] G. Stork, D. Niu, A. Fujimoto et al., "The first stereoselective total synthesis of quinine," Journal of the American Chemical Society, vol. 123, no. 14, pp. 3239-3242, 2001.

[3] I. T. Raheem, S. N. Goodman, and E. N. Jacobsen, "Catalytic asymmetric total syntheses of quinine and quinidine," Journal of the American Chemical Society, vol. 126, no. 3, pp. 706-707, 2004.

[4] S. Rollas and S. Güniz Küçükgüzel, "Biological activities of hydrazone derivatives," Molecules, vol. 12, no. 8, pp. 1910-1939, 2007.

[5] H. A. Saadeh, I. M. Mosleh, and M. S. Mubarak, "Synthesis of novel hybrid molecules from precursors with known antiparasitic activity," Molecules, vol. 14, no. 4, pp. 1483-1494, 2009.

[6] K. N. Dhawan, B. P. Jaju, and G. P. Gupta, "Validity of antagonism of different effects of reserpine as test for anti-depressant activity," Psychopharmacologia, vol. 18, no. 1, pp. 94-98, 1970.

[7] J. N. S. Yadava and G. P. Dutta, "Combined action of antiamoebic drugs and antibiotics on axenically grown Entamoeba histolytica," Indian Journal of Medical Research, vol. 61, pp. 971-975, 1973.

[8] S. S. Chakravarti, P. K. Sen, S. Choudhari, and M. Das, Indian Journal of Chemistry, vol. 248, p. 737, 1985.

[9] C. H. Browning, "The trypanocidal action of some derivatives of anil and styryl quinolines-II," Proceedings of the Royal Society of London B, vol. 768, pp. 372-377, 1932.

[10] C. H. Browning, "The antiseptic action of anil-pyridines and anil-quinolines," The Journal of Pathology and Bacteriology, vol. 27, pp. 121-122, 1924.

[11] B. M. Kotecka, G. B. Barlin, M. D. Edstein, and K. H. Rieckmann, "New quinoline di-mannich base compounds with greater antimalarial activity than chloroquine, amodiaquine, or pyronaridine?" Antimicrobial Agents and Chemotherapy, vol. 41, no. 6, pp. 1369-1374, 1997.

[12] L. A. Freek, J. L. Martin, Rowlett, and Willson, Journal of the American Chemical Society, vol. 68, p. 1285, 1946.

[13] R. J. Rowlett Jr. and R. E. Lutz, Journal of the American Chemical Society, vol. 68, pp. 1288-1191, 1946.

[14] I. V. Ukrainets, L. Yangyang, A. A. Tkach, and A. V. Turov, "4-Hydroxy-2-quinolones 166. Synthesis, isomerism, and antitubercular activity of 3-arylaminomethylene-quinoline-2,4$(1 \mathrm{H}, 3 \mathrm{H})$-diones," Chemistry of Heterocyclic Compounds, vol. 45 , no. 7, pp. 802-808, 2009.

[15] M. F. El-Zohry, A. E. H. N. Ahmed, F. A. Omar, and M. A. AbdAlla, "Synthesis and antibacterial activity of certain quinoline and quinazoline derivatives containing sulfide and sulfone moieties," Journal of Chemical Technology and Biotechnology, vol. 53, no. 4, pp. 329-336, 1992.

[16] C. H. Lee and H. S. Lee, "Relaxant effect of quinoline derivatives on histamine-induced contraction of the isolated guinea pig trachea," Journal of Applied Biological Chemistry, vol. 54, no. 1, pp. 118-123, 2011.

[17] H. Zheng, L. M. Weiner, O. Bar-Am et al., "Design, synthesis, and evaluation of novel bifunctional iron-chelators as potential agents for neuroprotection in Alzheimer's, Parkinson's, and other neurodegenerative diseases," Bioorganic and Medicinal Chemistry, vol. 13, no. 3, pp. 773-783, 2005.
[18] R. Michael, S. Ian, L. Paul et al., "3-Acyl-4-hydroxyquinolin$2(1 \mathrm{H})$-ones. Systemically active anticonvulsants acting by antagonism at the glycine site of the N-methyl-D-aspartate receptor complex," Journal of Medicinal Chemistry, vol. 36, no. 22, pp. 3386-3396, 1993, Chemical Abstracts, vol. 119, 249817y, 1993.

[19] M. P. Wentland, S. C. Aldous, M. D. Gruett et al., "The antitumor activity of novel pyrazoloquinoline derivatives," Bioorganic and Medicinal Chemistry Letters, vol. 5, no. 4, pp. 405-410, 1995.

[20] S. A. M. Bol, J. Horlbeck, J. Markovic, J. G. De Boer, R. J. Turesky, and A. Constable, "Mutational analysis of the liver, colon and kidney of Big Blue rats treated with 2-amino-3methlylimidazo[4,5-f] quinoline," Carcinogenesis, vol. 21, no. 1, pp. 1-6, 2000.

[21] H. I. El-Sabbagh, A. H. Abadi, I. E. Al-Khawad, and K. A. AlRashood, Archiv der Pharmazie, vol. 333, pp. 19-24, 1990.

[22] T. H. Althuis, P. F. Moore, and H. J. Hess, "Development of ethyl 3,4-dihydro-4-oxopyrimido[4,5-b]quinoline-2carboxylate, a new prototype with oral antiallergy activity," Journal of Medicinal Chemistry, vol. 22, no. 1, pp. 44-48, 1979.

[23] M. S. Pingle, S. P. Vartale, V. N. Bhosale, and S. V. Kuberkar, "A convenient synthesis of 3-cyano-4-imino-2-methylthio-4Hpyrimido [2,1-b] [1,3] benzothiazole and its reactions with selected nucleophiles," Arkivoc, vol. 2006, no. 10, pp. 190-198, 2006.

[24] O. M. Singh and M. F. Ahmed, "Cyclocondensation of cyanoketene dithioacetal with 2-aminopyridine: synthesis of pyrido [1, 2-a]pyrimidines," Indian Journal of Chemistry, vol. 43, no. 7, pp. 1561-1564, 2004.

[25] A. Z. M. Chowdhury and Y. Shibata, Chemical \& Pharmaceutical Bulletin, vol. 49, no. 4, p. 391, 2001.

[26] S. P. Vartale, V. N. Bhosale, S. V. Khansole, and R. N. Katapalle, "A convenient one pot synthesis of 3-cyano-9-methyl-2-methylthio-4-oxo- $4 \mathrm{H}$ - pyrimido[2, 1-b] pyrimido [4, 5-b] quinoline and its reactions with selected nucleophiles," Letters in Organic Chemistry, vol. 6, no. 7, pp. 544-548, 2009.

[27] R. Ananthanarayan and J. C. K. Panikar, Textbook of Microbiology, Orient Longman, 1999. 

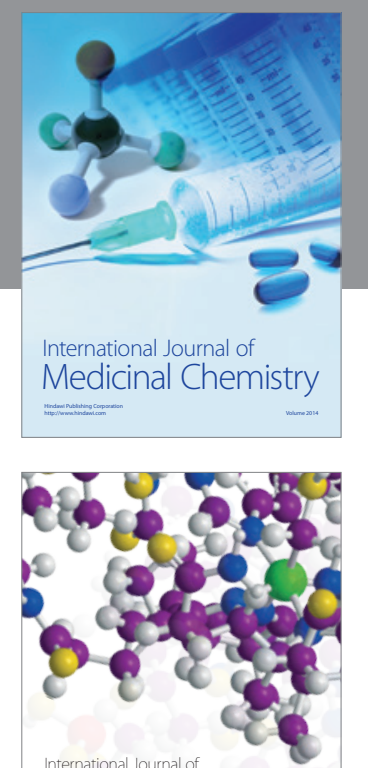

\section{Carbohydrate} Chemistry

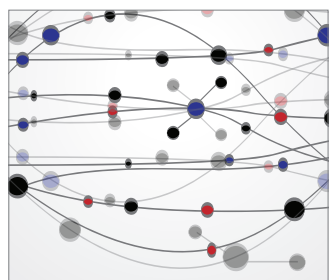

The Scientific World Journal
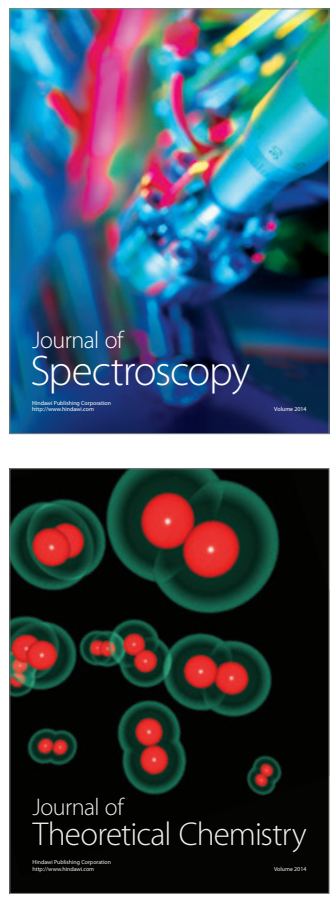
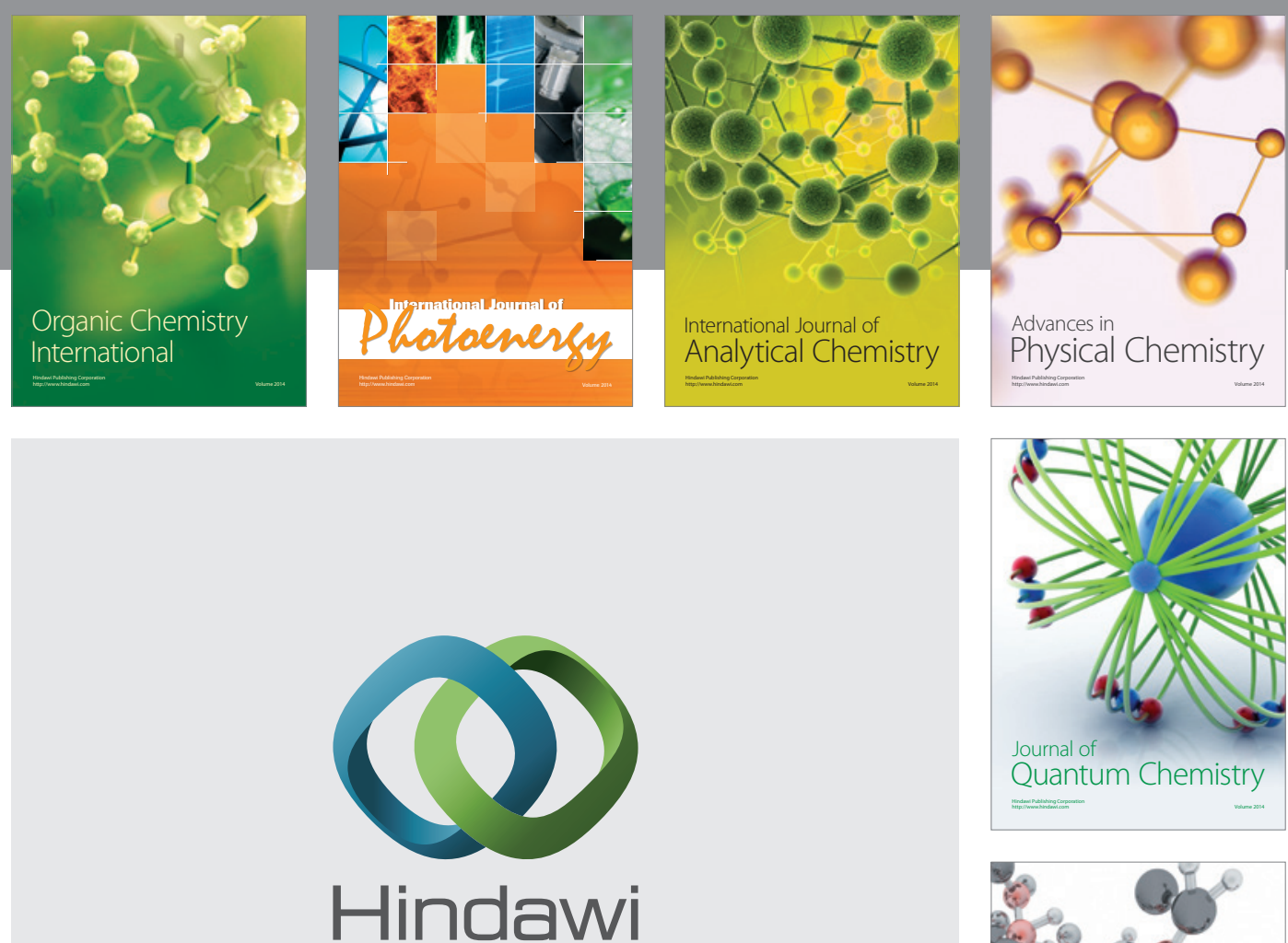

Submit your manuscripts at

http://www.hindawi.com

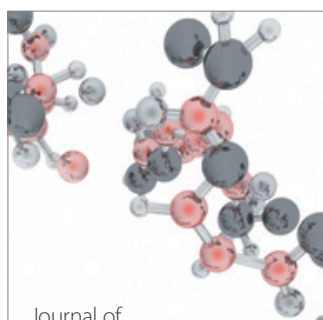

Analytical Methods

in Chemistry

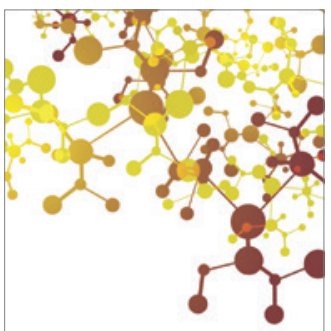

Journal of

Applied Chemistry

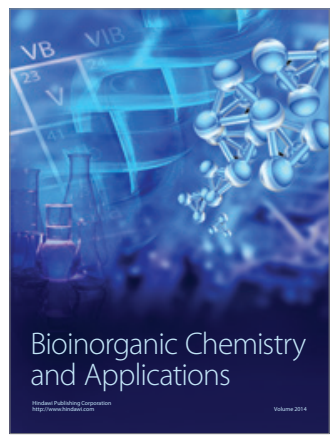

Inorganic Chemistry
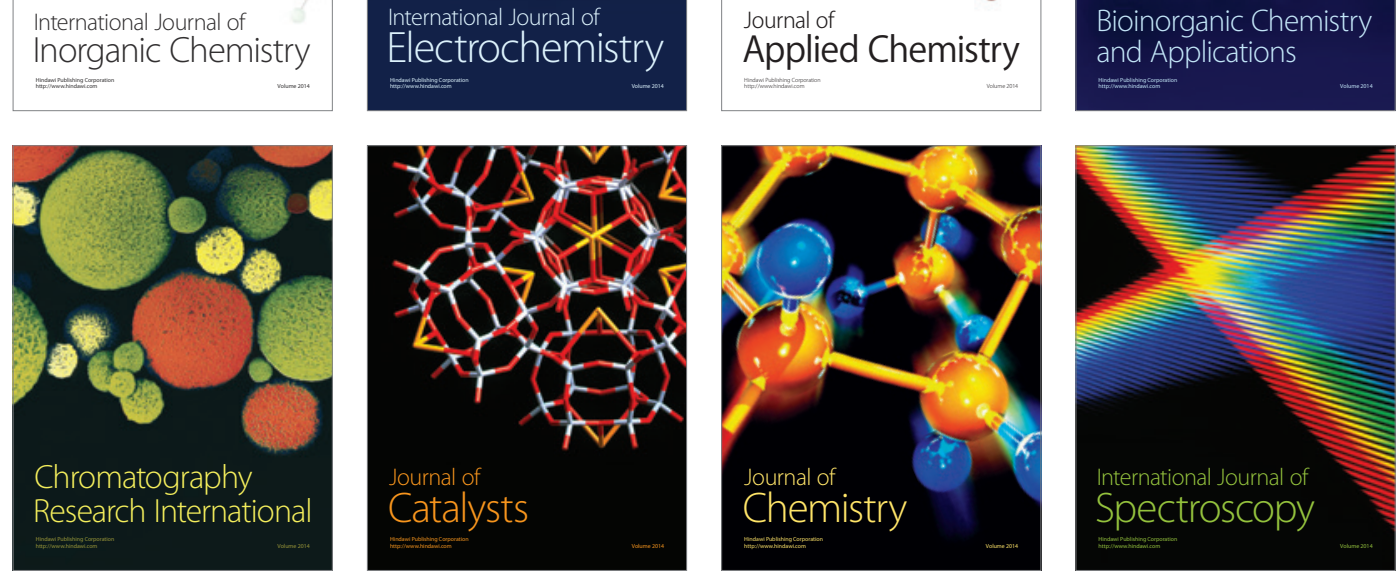